\title{
Cárie dentária em adolescentes da cidade de Recife- PE
}

\author{
Dental caries in adolescents of the city of Recife, PE, Brazil
}

\author{
Samara Sandrelly de Moura Gomes* \\ Silvia Regina Sampaio Bezerra** \\ Adriane Tenório Dourado** \\ Raíssa Peixoto de Arruda* \\ Agda Taís de Moura Silva*
}

\section{Resumo}

Objetivo: avaliar a presença de cárie dentária, dieta cariogênica, fatores socioeconômicos, demográficos e hábitos de higiene bucal em adolescentes da cidade de Recife- PE. Sujeitos e método: foi realizado um estudo transversal, qualitativo, que utilizou como instrumento de pesquisa um formulário para coleta de dados e uma ficha clínica. Foi empregada uma amostra por conveniência de 10 pacientes das clínicas de hebiatria, com idades entre 13 e 16 anos, de ambos os sexos. Resultados: $60 \%$ dos pacientes analisados tiveram cárie dentária e 40\% eram desprovidos de cárie. Aqueles cujas famílias possuem renda familiar entre 3 e 4 salários mínimos ou de 4 a 7 salários, obtiveram média de cárie dentária elevada: 0,60 e 1,20, respectivamente. $O$ aumento da média de dentes cariados foi maior entre os que escovavam três vezes ao dia do que entre aqueles que escovavam duas vezes ao dia. A cárie dentária reduziu à medida que o pesquisado fazia maior consumo de açúcar e aumentou quando este fazia o uso de fio dental de maneira mais frequente. Conclusões: na amostra estudada, a frequência da cárie dentária, o consumo de açúcar, os fatores socioeconômicos, hábitos de higiene bucal e gênero feminino foram considerados elevados.

Palavras-chave: Cárie dentária. Epidemiologia. Dieta cariogênica.

\section{Introdução}

A doença cárie é caracterizada como um desequilíbrio no processo de desmineralização e remineralização, resultante do acúmulo bacteriano e do seu metabolismo na superfície dentária. Com o surgimento de novos conceitos a respeito desta doença, a detecção, principalmente em seus estágios iniciais, vem se tornando um processo cada vez mais comple$\mathrm{xo}^{1}$. Apesar de ser um estudo feito no mundo inteiro, à maioria das pesquisas concentra-se em crianças em idade escolar, não havendo dados suficientes na literatura sobre a prevalência de cárie dentária em adolescentes ${ }^{2}$.

A adolescência é tida como um período de maior comportamento de risco para cárie dentária, em decorrência do precário controle de placa e da redução dos cuidados com a higiene bucal, agravados pela maior independência em relação ao consumo de uma alimentação mais açucarada ${ }^{3}$. A juventude, de modo geral, tem se mostrado com o mesmo comportamento alimentar por todo o mundo, ainda que seja proveniente de culturas e países diversos, pois já aderiu à inclusão de refrigerantes, suco de frutas, café e leite com adição de açúcar na dieta diária ${ }^{4-5}$. Um estudo transversal realizado com adolescentes de uma escola pública de Muriaré (Minas Gerais), para análise

Acadêmica de graduação em Odontologia, Faculdade de Odontologia da Universidade Pernambuco - FOP/UPE, Recife - PE, Brasil.

Professora Doutora Adjunta da disciplina de Clínica de Alta e Média Complexidade, Faculdade de Odontologia da Universidade de Pernambuco - FOP/UPE, Recife - PE, Brasil. 
dos hábitos alimentares, observou que grande parte dos adolescentes possuía costumes alimentares inadequados, como um elevado consumo de alimentos ricos em gorduras, doces e refrigerantes, e um baixo consumo diário de frutas, hortaliças, leite e derivados. Além disso, observou-se um alto índice de substituições das principais refeições por lanches ${ }^{6}$.

Os hábitos e estilo de vida influenciam a condição de saúde bucal, ao passo que a perda dentária é alta, principalmente pela cárie dentária. Quanto maior a prevalência e severidade da cárie dentária em adolescentes, menor o grau de escolaridade da mãe e menor o número de consultas ao cirurgião-dentista ${ }^{7}$.

Pesquisas feitas em 2005 mostraram a relação entre posição socioeconômica e as condições de saúde. Indivíduos que ocupam posição superior na hierarquia social apresentam melhores condições de saúde que os indivíduos de posições imediatamente inferiores ${ }^{8}$. Embora exista aumento na utilização de serviços odontológicos em todas as camadas sociais no Brasil, essa utilização ainda é muito desigual, pois a proporção de pessoas que nunca visitaram serviços odontológicos é oito vezes maior nos mais pobres ${ }^{9}$.

Estudos realizados em 2016 mostraram o uso de telas de computadores e televisões, consumo de refeições e petiscos por adolescentes brasileiros e atentaram que esse hábito foi maior em adolescentes de escolas públicas. Isso pode estar relacionado ao nível de instrução e à rotina de trabalho dos pais, uma vez que, para a realização das refeições à mesa e para o incentivo à prática de atividade física, o apoio familiar é importante, tanto quanto a conscientização dos próprios adolescentes. As telas de computadores e televisões têm ocupado lugar de destaque no âmbito familiar, levando a profundas mudanças no estilo de vida da população $0^{10}$.

Pesquisas com 215 adolescentes (15-19 anos) foram realizadas para estimar a condição de saúde bucal e verificar a existência de impacto na qualidade de vida, e verificou-se que a maioria desses adolescente mora com os pais, cursaram o ensino fundamental em uma instituição pública de ensino, possui mães que concluíram o ensino médio e suas famílias recebem entre dois e três salários mínimos de renda mensal bruta. Em relação à qualidade de vida relacionada à saúde bucal, pouco mais de $50 \%$ dos adolescentes relataram na pesquisa dificuldade para realizar ao menos uma atividade diária em decorrência de problemas odontológicos, portanto evidenciaram impacto em sua qualidade de vida ${ }^{11}$.

A frequência da escovação encontra-se associada ao nível escolar dos pais, registrando-se diferenças estatisticamente significativas entre os vários estudos analisados. Os adolescentes cujos pais apresentam uma escolaridade inferior a 4 anos, referem menor frequência de escovação. Nestes adolescentes, $22,6 \%$ referem escovar menos de uma vez por dia, 59,2\% uma vez por dia e apenas $18,2 \%$ duas ou mais vezes por dia ${ }^{12}$.
A educação é associada com a maior periodicidade de escovação; indivíduos com maior nível de escolaridade e melhor nível socioeconômico escovam mais os dentes e a maior porcentagem dos usuários de creme dental fluoretado pertence ao grupo de renda mais alta ${ }^{13}$

Neste contexto, este estudo objetiva determinar se existe relação entre cárie dentária, dieta cariogênica e perfil socioeconômico, demográfico e hábitos de higiene bucal, em adolescentes dependentes de Militares da cidade de Recife PE.

\section{Sujeitos e método}

Trata-se de um estudo transversal, qualitativo, realizado no período de outubro a novembro de 2016, em uma clínica de um serviço militar pertencente à cidade de Recife - PE, município brasileiro localizado na região nordeste do país. Foi utilizada uma amostra de conveniência de 10 adolescentes, constituída por jovens adolescentes dependentes de Militares de ambos os gêneros de 13 a 16 anos, a fim de servir de base para futuros estudos. O processo de calibração foi avaliado através da concordância intraexaminador para o diagnóstico do componente "C" do índice CPO-D, segundo os critérios preconizados pela Organização Mundial de Saúde (OMS) em sua quarta versão (1997), com o autor da pesquisa.

No primeiro momento, foi realizado o contato inicial com o paciente, pai e/ou responsável, sendo explicada a finalidade da pesquisa, seu objetivo, a relevância do estudo e sendo assim, solicitada a participação do entrevistado e assinatura do Termo de Consentimento Livre e Esclarecido e do Termo de Assentimento. Em seguida, foi realizada uma entrevista, em que o pesquisador utilizou o método do interrogatório cruzado, conduzindo as perguntas através de um formulário. A coleta dos dados foi constituída de três etapas: identificação geral do paciente e dos aspectos demográficos (idade, gênero e etnia) contidos na ficha clínica, questões sobre aspectos socioeconômicos, hábitos de higiene bucal e alimentação. O formulário continha 12 perguntas fechadas e abertas. Em uma terceira etapa foi realizada a coleta de dados nos pacientes através do exame físico intrabucal, para a determinação do componente "C" (cariado) do índice CPO-D, utilizando-se conjuntos compostos por espelho bucal plano e sonda exploradora de ponta romba, ambos esterilizados, e o pesquisador com o Equipamento de Proteção Individual (EPI) adequado ao ambiente de trabalho, seguindo as normas de biossegurança do Ministério da Saúde para levantamento epidemiológico. Os dados foram anotados no momento do exame em uma ficha clínica específica contendo dados referentes à condição dental do paciente.

Os dados foram analisados descritivamente através de frequências absolutas e percentuais 
para as variáveis categóricas e as medidas: (média e desvio padrão para as variáveis numéricas) e foram utilizados inferencialmente através dos testes estatísticos. Para avaliar associação entre duas variáveis categóricas foi utilizado o teste Exato de Fisher; para a comparação do número de dentes cariados entre duas categorias foi utilizado o teste de Mann-Whitney e na comparação de três ou mais categorias, foi utilizado o teste de Kruskal-Wallis.

Esse projeto foi encaminhado ao Comitê de ética em Pesquisa da Universidade de Pernambuco, onde foi considerado aprovado em 16/09/2016.

\section{Resulltados}

A maioria dos avaliados era de etnia branca e tinha quatro pessoas residentes em casa. A renda familiar dos pesquisados teve média de 4,14 salários mínimos, desvio padrão de 1,07 e mediana igual a 3,75 salários mínimos. $\mathrm{O}$ maior percentual de pesquisados morava em residência própria, nove mães eram do lar e todos os pais eram militares. Apenas a família de um pesquisado não tinha automóvel. Na Tabela 1 se apresentam os resultados das características socioeconômicas e demográficas dos pesquisados.

Tabela 1 - Características socioeconômicas e demográficas da amostra estudada

\begin{tabular}{|c|c|c|}
\hline Variável & $\mathrm{n}$ & $\%$ \\
\hline TOTAL & 10 & 100,0 \\
\hline \multicolumn{3}{|l|}{ Idade } \\
\hline 13 anos & 4 & 40,0 \\
\hline 14 anos & 3 & 30,0 \\
\hline 15 anos & 1 & 10,0 \\
\hline 16 anos & 2 & 20,0 \\
\hline \multicolumn{3}{|c|}{ Renda familiar (Salários mínimos) } \\
\hline Entre 3 e 4 & 5 & 50,0 \\
\hline Mais de 4 a 7 & 5 & 50,0 \\
\hline \multicolumn{3}{|l|}{ Etnia } \\
\hline Branca & 7 & 70,0 \\
\hline Parda & 1 & 10,0 \\
\hline Negra & 2 & 20,0 \\
\hline \multicolumn{3}{|c|}{ Número de pessoas morando em casa } \\
\hline 4 & 6 & 60,0 \\
\hline 5 & 3 & 30,0 \\
\hline 6 & 1 & 10,0 \\
\hline \multicolumn{3}{|l|}{ Moradia } \\
\hline Própria & 7 & 70,0 \\
\hline Alugada & 3 & 30,0 \\
\hline \multicolumn{3}{|l|}{ Ocupação da mãe } \\
\hline Do lar & 9 & 90,0 \\
\hline Gerente de loja & 1 & 10,0 \\
\hline \multicolumn{3}{|l|}{ Ocupação do pai } \\
\hline Militar & 10 & 100,0 \\
\hline \multicolumn{3}{|l|}{ Posse de automóvel } \\
\hline Não possui & 1 & 10,0 \\
\hline Possui um automóvel & 6 & 60,0 \\
\hline Possui dois automóveis & 3 & 30,0 \\
\hline
\end{tabular}

Considerando os parâmetros da avaliação dos hábitos de higiene bucal e dieta, ressalta-se que a maioria $(70,0 \%)$ declarou fazer três escovações por dia e (30\%) escovavam duas vezes ao dia. As frequências dos que consumiam açúcar uma vez ao dia, duas vezes ao dia e três vezes ao dia foram 3,4 e 3 respec- tivamente. Todos utilizaram flúor profissional, sendo $70,0 \%$ em gel, 10,0\% em forma de bochecho e $20,0 \%$ de ambas as formas. As frequências dos que não utilizavam fio dental, utilizavam uma vez ou duas vezes foram 4, 3 e 3 respectivamente (Tabela 2 ).

Tabela 2 - Avaliação das variáveis de hábitos de higiene bucal e dieta

\begin{tabular}{l|r|c}
\hline \multicolumn{1}{|c|}{ Variável } & $\mathrm{n}$ & $\%$ \\
\hline TOTAL & 10 & 100,0 \\
\hline Frequência de escovação dentária & & \\
$\quad$ Duas vezes ao dia & 3 & 30,0 \\
Três vezes ao dia & 7 & 70,0 \\
Frequência de consumo de açúcar na dieta & & \\
alimentar & & \\
Uma vez ao dia & 3 & 30,0 \\
Duas vezes ao dia & 4 & 40,0 \\
Três vezes ao dia & 3 & 30,0 \\
Utilização de flúor profissional & & \\
Em forma de gel & 7 & 70,0 \\
Bochechos & 1 & 10,0 \\
Ambas as formas & 2 & 20,0 \\
Frequência de uso de fio dental & & \\
Nenhuma vez & 4 & 40,0 \\
Uma vez ao dia & 3 & 30,0 \\
Duas vezes ao dia & 3 & 30,0 \\
Quantidade de creme dental usada quando & & \\
criança & & \\
Foto 1 & 1 & 10,0 \\
Foto 2 & 1 & 10,0 \\
Foto 3 & 4 & 40,0 \\
Foto 4 & 4 & 40,0 \\
Quantidade de creme dental usada quando & & \\
adolescente & & \\
Foto 1 & - & - \\
Foto 2 & 1 & 10,0 \\
Foto 3 & 2 & 20,0 \\
Foto 4 & 7 & 70,0 \\
\hline
\end{tabular}

Tabela 3 mostra que quatro adolescentes não tinham cárie dentária e quatro tinham uma cárie. Dois adolescentes apresentavam duas ou três cáries. Desta forma, conclui-se que $60,0 \%$ dos pesquisados tinham cárie dentária e 40,0\% não tinham.

Tabela 3 - Avaliação da cárie dentária

\begin{tabular}{l|r|c}
\hline \multicolumn{1}{c|}{ Variável } & $\mathrm{n}$ & $\%$ \\
\hline TOTAL & 10 & 100,0 \\
Número de dentes com cárie & & \\
Nenhum & 4 & 40,0 \\
Um & 4 & 40,0 \\
Dois & 1 & 10,0 \\
Três & 1 & 10,0 \\
Cárie dentária & & \\
Com & 6 & 60,0 \\
Sem & 4 & 40,0 \\
\hline
\end{tabular}

Na Tabela 4 é apresentado os resultados do estudo da associação entre cárie dentária com cada uma das variáveis socioeconômica, demográfica, hábitos de higiene bucal e dieta. Embora algumas frequências percentuais com cárie tenham se mostrado com diferenças elevadas para a margem de erro fixada (5\%), não se comprova diferença significativa ( $p>0,05)$ entre a presença ou ausência de cárie e as variáveis socioeconômica, demográfica, hábitos de higiene bucal e dieta. 
Tabela 4 - Presença ou ausência de cárie segundo as variáveis socioeconômica, demográfica, hábitos de higiene bucal e dieta

\begin{tabular}{|c|c|c|c|c|c|c|c|}
\hline \multirow{3}{*}{ Variável } & \multicolumn{4}{|c|}{ Cárie dentária } & \multirow{2}{*}{\multicolumn{2}{|c|}{ TOTAL }} & \multirow{3}{*}{ Valor de $\mathrm{p}$} \\
\hline & \multicolumn{2}{|c|}{ Sim } & \multicolumn{2}{|c|}{ Não } & & & \\
\hline & $\mathrm{n}$ & $\%$ & $\mathrm{n}$ & $\%$ & $\mathrm{n}$ & $\%$ & \\
\hline TOTAL & 6 & 60,0 & 4 & 40,0 & 10 & 100,0 & \\
\hline Gênero & & & & & & & $\mathrm{p}^{(1)}=0,190$ \\
\hline Masculino & 1 & 25,0 & 3 & 75,0 & 4 & 100,0 & \\
\hline Feminino & 5 & 83,3 & 1 & 16,7 & 6 & 100,0 & \\
\hline Número de residentes em casa & & & & & & & $\mathrm{p}^{(1)}=1,000$ \\
\hline 4 pessoas & 4 & 66,7 & 2 & 33,3 & 6 & 100,0 & \\
\hline 5 ou 6 pessoas & 2 & 50,0 & 2 & 50,0 & 4 & 100,0 & \\
\hline Moradia & & & & & & & $\mathrm{p}^{(1)}=0,500$ \\
\hline Própria & 5 & 71,4 & 2 & 28,6 & 7 & 100,0 & \\
\hline Alugada & 1 & 33,3 & 2 & 66,7 & 3 & 100,0 & \\
\hline Renda familiar (Salários mínimos) & & & & & & & $\mathrm{p}^{(1)}=1,000$ \\
\hline Entre 3 e 4 & 3 & 60,0 & 2 & 40,0 & 5 & 100,0 & \\
\hline Mais de 4 a 7 & 3 & 60,0 & 2 & 40,0 & 5 & 100,0 & \\
\hline Frequência de escovação dentária & & & & & & & $\mathrm{p}^{(1)}=1,000$ \\
\hline Duas vezes ao dia & 2 & 66,7 & 1 & 33,3 & 3 & 100,0 & \\
\hline Três vezes ao dia & 4 & 57,1 & 3 & 42,9 & 7 & 100,0 & \\
\hline Frequência consumo de açúcar na dieta & & & & & & & $\mathrm{p}^{(1)}=0,743$ \\
\hline Uma vez ao dia & 2 & 66,7 & 1 & 33,3 & 3 & 100,0 & \\
\hline Duas vezes ao dia & 3 & 75,0 & 1 & 25,0 & 4 & 100,0 & \\
\hline Três vezes ao dia & 1 & 33,3 & 2 & 66,7 & 3 & 100,0 & \\
\hline Frequência do uso de fio dental & & & & & & & $\mathrm{p}^{(1)}=0,400$ \\
\hline Nenhuma vez & 2 & 50,0 & 2 & 50,0 & 4 & 100,0 & \\
\hline Duas vezes ao dia & 1 & 33,3 & 2 & 66,7 & 3 & 100,0 & \\
\hline Três vezes ao dia & 3 & 100,0 & - & - & 3 & 100,0 & \\
\hline
\end{tabular}

(1) Através do teste Exato de Fisher.

Não foram registradas diferenças significativas ( $p>0,05)$ entre as categorias em relação ao número de cáries. Entretanto, destaca-se que as médias do número de cáries foram correspondentemente mais elevadas entre os pesquisados do gênero feminino do que no gênero masculino $(1,17 \times 0,50)$; entre os que tinham 4 residentes do que entre os que tinham 5 a 6 residentes $(1,17 \times 0,50)$; entre os que tinham casa própria do que alugada $(1,14 \times 0,33)$; entre os que tinham renda familiar de 4 a 7 salários mínimos do que entre os que tinham renda familiar entre 3 e 4 salários mínimos $(1,20 \times 0,60)$; entre os que escovavam os dentes três vezes do que entre os que escovavam duas vezes ao dia $(1,00 \times 0,67)$. A cárie dentária reduziu à medida que o pesquisado fazia maior consumo de açúcar; aumentou à medida que o pesquisado fazia o uso de fio dental de maneira mais frequente, conforme resultados apresentados na Tabela 5.
Tabela 5 - Estatística do número de dentes cariados segundo as variáveis socioeconômica, demográfica e hábitos de higiene bucal e dieta

\begin{tabular}{|c|c|c|c|}
\hline Variável & Média & $\begin{array}{l}\text { Desvio } \\
\text { padrão }\end{array}$ & Valor $p$ \\
\hline \multicolumn{4}{|l|}{ Sexo } \\
\hline Masculino $(n=4)$ & 0,50 & 1,00 & \multirow[t]{2}{*}{$p^{(1)}=0,243$} \\
\hline Feminino $(n=6)$ & 1,17 & 0,98 & \\
\hline \multicolumn{4}{|l|}{ № de residentes } \\
\hline $4(n=6)$ & 1,17 & 1,17 & \multirow[t]{2}{*}{$p^{(1)}=0,438$} \\
\hline 5 a $6(n=4)$ & 0,50 & 0,58 & \\
\hline \multicolumn{4}{|l|}{ Moradia } \\
\hline Própria $(\mathrm{n}=7)$ & 1.14 & 1,07 & \multirow[t]{2}{*}{$\mathrm{p}^{(1)}=0,400$} \\
\hline Alugada $(\mathrm{n}=3)$ & 0,33 & 0,58 & \\
\hline \multicolumn{4}{|c|}{ Renda familiar (Salários mínimos) } \\
\hline Entre 3 e $4(n=5)$ & 0,60 & 0,55 & \multirow[t]{2}{*}{$p^{(1)}=0,683$} \\
\hline Mais de 4 a $7(n=5)$ & 1,20 & 1,30 & \\
\hline \multicolumn{4}{|l|}{ Frequência escovação dentária } \\
\hline Duas vezes ao dia $(n=3)$ & 0,67 & 0,58 & \multirow[t]{2}{*}{$p^{(1)}=1,00$} \\
\hline Três vezes ao dia $(n=7)$ & 1,00 & 1,16 & \\
\hline \multicolumn{4}{|l|}{$\begin{array}{l}\text { Frequência consumo de açúcar } \\
\text { na dieta }\end{array}$} \\
\hline Uma vez ao dia $(\mathrm{n}=3)$ & 1,33 & 1,53 & \multirow[t]{3}{*}{$\mathrm{p}^{(2)}=0,475$} \\
\hline Duas vezes ao dia $(n=4)$ & 1,00 & 0,82 & \\
\hline Três vezes ao dia $(n=3)$ & 0,33 & 0,58 & \\
\hline \multicolumn{4}{|l|}{ Frequência do uso de fio dental } \\
\hline Nenhuma vez $(n=4)$ & 0,50 & 0,58 & \multirow[t]{3}{*}{$\mathrm{p}^{(2)}=0,286$} \\
\hline Uma vezes ao dia $(n=3)$ & 0,67 & 1,16 & \\
\hline Duas vezes ao dia $(n=3)$ & 1,67 & 1,15 & \\
\hline
\end{tabular}

(1) Através do teste de Mann-Whitney

(2) Através do teste de Kruskal-Wallis. 


\section{Discussão}

No total de pesquisados com cárie dentária, verificou-se que mais da metade possuía dentes com cárie. Tal associação é explicada por um estudo que revelou que os usuários do serviço público pertencem a uma população economicamente menos favorecida e com menor acesso ao atendimento odontológico, sugerindo a necessidade de garantir a prevenção e controle da cárie e o acesso a tratamentos dentários restauradores no serviço público ${ }^{14}$. Apesar da reconhecida importância da saúde bucal, uma parcela importante da população brasileira não utiliza os serviços odontológicos frequentemente ${ }^{15}$.

O número de cáries foi ressaltado nas famílias que possuíam maior renda (entre de 4 e 7 salários mínimos), divergindo dos resultados encontrados em outro estudo, o qual relatou que quem dispõe de uma renda menor e menos bens foram os que mostraram possuir maior chance de ter um alto índice de cárie ${ }^{16}$. Uma possível justificativa para este dissentimento seria a ausência de inspeção dos pais perante os filhos em relação à higiene bucal, acreditando que estes a fazem corretamente e todos os dias, sem precisarem ser repreendidos ou ensinados.

Nos resultados encontrados, observou-se que a cárie dentária foi elevada na presença de menos residentes morando em casa, divergindo de pesquisa realizada no ano de 2011, na qual constatou-se que uma moradia em domicílios com seis ou mais residentes, mais de três pessoas por quarto e tempo de moradia na área de três ou mais anos, estiveram estatisticamente associados à experiência de cárie dentária ${ }^{17}$. Tal resultado pode ser explicado devido que em tempos atuais a quantidade de filhos, por menor que seja, não afeta na diminuição da presença de cárie.

$\mathrm{Na}$ grande maioria das famílias o processo de aquisição dos hábitos de higienização bucal inicia-se cedo na infância, mas com os problemas sociais encontrados por algumas famílias, essa atitude às vezes passa despercebida pelos responsáveis, que deveriam ao menos realizar as primeiras escovações dos filhos, sendo que em muitos dos casos, os filhos imitam os atos realizados pelos mais velhos ${ }^{18}$. No que diz respeito à ocupação dos pais observados nesta pesquisa, a maioria das mães eram do lar $(90 \%)$ e os pais militares, logo, pode-se presumir que as mães passariam mais tempo com os filhos em casa e poderiam fiscalizar e ensinar uma melhor escovação bucal e controle da dieta cariogênica. As práticas diárias de escovação e o controle da dieta são cuidados importantes em relação à saúde bucal.

A cárie dentária aumentou em pesquisados que escovavam os dentes mais vezes ao dia, e reduziu à medida que se fazia o maior consumo de açúcar. Uma justificativa plausível, seria que, por estarem sentados em uma cadeira odontológica, diante dos pais e um dentista, os adolescentes pesquisados sentir-se-iam embaraçados e coagidos em responder o questionário corretamente, com receio de existir alguma repreensão pelos pais e/ou dentista, mediante as respostas obtidas. Podemos justificar essa análise de comportamento, uma elevada exposição às telas entre escolares de escolas particulares. Isso pode ser consequência de os estudantes de escolas privadas terem mais acesso aos avanços tecnológicos, como os elétricos e eletrônicos e, assim, ficarem mais tempo em uso desses equipamentos ${ }^{19}$.

Um dos métodos mais utilizados para a manutenção de um constante nível de flúor no meio bucal é o creme dental. Em muitos países de baixa renda, o creme dental com flúor é provavelmente a melhor maneira para população no controle e na prevenção da cárie dentária ${ }^{20}$. Pelos dados pesquisados referentes à quantidade de creme dental usada quando criança e adolescente constata-se que em se tratando da quantidade, houve prevalência pelo completo preenchimento das cerdas das escovas, o que não se pode reiterar em pesquisas feitas por um determinado estudo ${ }^{21}$. Supõe-se que os pais das crianças atendidas na clínica não supervisionavam e não participavam da escovação dos dentes de seus filhos, acreditando que eles escovavam os dentes corretamente, ou, os pais não compreendem a quantidade de creme dental precisa. Assegura isso pesquisa realizada em 2006, na qual alguns pais explicam que a causa da pouca participação nesse cuidado em casa está associada ao trabalho ou mesmo até por desconhecerem as técnicas corretas de escovação ${ }^{22}$.

A resistência ao uso do fio dental é observada entre os jovens. Eles têm dificuldade em seu uso pela habilidade requerida, pois necessita de treino, e possuem preguiça em adotá-lo como recurso de rotina $^{23}$. Os dados obtidos na pesquisa ressaltam que $40 \%$ dos pesquisados não utilizam fio dental e $30 \%$ utilizam pelo menos duas vezes ao dia. Tais achados também foram semelhantes em outro estudo, no qual apenas $20 \%$ usavam fio dental regularmente ${ }^{24}$.

Análises feitas com adolescentes para avaliar a influência do gênero na prevalência de cárie dentária, verificaram a associação entre o gênero e a higiene bucal, sendo que os meninos apresentaram uma prevalência de placa dental maior do que as meninas $(p<0,001)$. Além disso, eles apresentaram uma maior prevalência de relato de frequência diária de escovação, menor do que duas vezes ao dia, quando comparados com as meninas ${ }^{25}$. Contrastando a essas análises, o número de cárie mais elevado nesta pesquisa é representado pelo sexo feminino. A adolescência é uma fase de grandes riscos para problemas de saúde bucal, tais como cárie, gengivite, recessão e sangramento gengival. Fatores sociodemográficos, psicossociais e o estilo de vida adotado pelo indivíduo determinam os hábitos de higiene e a própria saúde ${ }^{26}$.

Os adolescentes que participaram dessa pesquisa foram acompanhados por seus pais durante 
o exame clínico e por se tratar de uma amostra de conveniência, não serão apresentadas conclusões probabilísticas de inferência populacional para 0 município. Porém, os resultados apresentados deverão servir de base para futuros estudos mais abrangentes e conclusivos que serão realizados. Neste contexto, mais pesquisas serão necessárias para confirmar a frequência e fatores relacionados a cárie dentária na população estudada.

\section{Conclusões}

De acordo com os resultados encontrados, pode-se concluir que:

a) A frequência de cárie dentária dos adolescentes foi alta;

b) O consumo de açúcar e fator socioeconômico foram altos, assim como os hábitos de higiene bucal;

c) A cárie dentária foi mais elevada no gênero feminino;

d) Não houve associação significativa entre as variáveis socioeconômicas, demográficas, hábitos de higiene bucal e alimentação com a presença da cárie dentária.

\section{Abstract}

Objective: to evaluate the presence of dental caries, cariogenic diet, socioeconomic and demographic factors, and oral hygiene habits in adolescents of the city of Recife, Pernambuco, Brazil. Subjects and method: a qualitative cross-sectional study was performed using a data collection form and a clinical record as research instruments. A convenience sample was applied, including 10 patients of hebiatrics clinics, aged 13 to 16 years, of both genders. Results: $60 \%$ of the patients examined presented dental caries and $40 \%$ did not. Those with family incomes between 3 and 4 minimum wages or 4 and 7 wages obtained high means of dental caries: 0.60 and 1.20, respectively. The increase in means of dental caries was higher among those who brushed their teeth three times a day than those who brushed twice a day. Dental caries decreased as the subjects consumed more sugar and increased as they used dental floss more often. Conclusions: in the sample studied, the frequency of dental caries, sugar consumption, socioeconomic factors, oral hygiene habits, and female gender were considered high.

Keywords: Dental caries. Epidemiology. Cariogenic diet.

\section{Referências}

1. Alencar AA, Queiroz NO, Lima HT, Quirino ABG, Feitosa VP. Métodos alternativos de detecção de cárie: uma revisão de literatura. Jornada Odontológica dos Acadêmicos da Católica; 2016.

2. Gonçalves ER, Peres MA, Marcenes W. Cárie dentária e condições sócio- econômicas: um estudo transversal com jovens de 18 anos de Florianópolis, Santa Catarina, Brasil. Cad Saúde Pública 2002;18(3):699-706.

3. Garbin, CAS, Moreira AR, Saliba NA, Goncalves PE. La salud bucal en la percepción del adolescente. Rev Salud Publica $2009 ; 11(2): 268-77$.

4. Feldens CA, Rodrigues PH, Rauber F, Chaffee BW, Vitolo MR Food expenditures, cariogenic dietary practices and childhood dental caries in southern Brazil. Caries Research 2013; 47(5):373-81.

5. Guido JA, Martinez EA, Soto A, Eggertsson H, Sanders BJ, Jones JE, et al. Caries prevalence and its association with brushing habits, water availability, and the intake of sugared beverages. Int Paediatric Dentistry 2011; 21(6):432-40.

6. Resende FR, Fófano PBR Nunes GR, Vieira TG, Quintão DF. Análise dos hábitos alimentares e das práticas de higiene de adolescentes de uma escola pública de Muriaé (MG). Ver Cient FAMINAS, 2015; 11(1).

7. Traebert J, Jinbo Y, \& de Lacerda JT.Association between maternal schooling and caries prevalence: a cross-sectional study in southern Brazil. Oral Health and Preventive Dentistry, 2011; 9(1):47.

8. Marmot M. Historical perspective: the social determinants of disease-some blossoms. Epidemiologic perspectives \& innovation, 2005; EP+ I, 2, 4.

9. Peres KG, Peres MA, Boing AF, Bertoldi AD, Bastos JL, Barros AJ. Reduction of social inequalities in utilization of dental care in Brazil from 1998 to 2008. Rev de Saude Publica 2012; 46(2):250-8.

10. Oliveira JS, Barufaldi LA, de Azevedo Abreu G, Leal VS, Brunken GS, et al. ERICA: uso de telas e consumo de refeições e petiscos por adolescentes brasileiros. Rev de Saúde Pública 2016; 50(suppl. 1), 7.

11. Filgueira ACG, Machado FCDA, Amaral BA, Lima K, Assunção I. Saúde bucal de adolescentes escolares, 2016.

12. Pereira C, Veiga N, Amaral O, Pereira J. Comportamentos de saúde oral em adolescentes portugueses. Rev Portuguesa de Saúde Pública, 2013; 31(2):145-52.

13. Tseveenjav B, Suominen-Taipale L, Varsio S, Hausen H, Knuuttila M, Vehkalahti MM. Patterns of oral cleaning habits and use of fluoride among dentate adults in Finland. Oral health \& preventive dentistry 2009; 8(3):287-94.

14. Silveira FM, Silveira FR, Nepomuceno MO, Martins EBL, Marcopito LF. Cárie dentária e fatores associados entre adolescentes no norte do estado de Minas Gerais, Brasil: uma análise hierarquizada. Rev Ciência \& Saúde Colet 2015; 20(11):

15. Gibilini C, Campos CEE, Volpato LF, Meneghim ZMDAP, da Silva DD, de Sousa MDLR. Acesso a serviços odontológicos e auto-percepcão da saúde bucal em adolescentes, adultos e idosos. Arq odontologia 2016; 46(4):

16. Da Silva MDFC, Residência Multiprofissional em saúde coletiva 2013.

17. Melo MMDCD, Souza WVD, Lima MLCD, Braga C.Fatores associados à cárie dentária em pré-escolares do Recife, Pernambuco, Brasil. Cad Saúde Pública 2011; 471-85.

18. Modena CM. Ciência e saúde coletiva 2005; 10 (1):

19. Oliveira TCD, Silva AAMD, Santos CDJND, Silva JS, Conceição SIOD. Atividade física e sedentarismo em escolares 
da rede pública e privada de ensino em São Luís. Rev de Saude Publica 2010; 44(6):996-1004.

20. Scabar LF, Manfedini MA, Armonia PL, Narvai PC, Frazão P. Frequência de uso de creme dental segundo renda e escolaridade: uma revisão sistemática, 2014; 318-25.

21. Evans DJ. A study of developmental defects in enamel in 10 -year-old high social class children residing in a non-fluoridated area. Community Dental Health 1991; 8(1):31-8.

22. Brasil MS. Secretaria de atenção à saúde. Departamento da atenção básica. Saúde Bucal/Ministério da saúde, Secretaria de atenção à saúde- Brasília: MS; 2006.

23. Flores EMTL, Drehmer TM. Conhecimentos, percepções, comportamentos e representações de saúde e doença bucal dos adolescentes de escolas públicas de dois bairros de Porto Alegre. Ciência \& Saúde Coletiva 2003; 8(3):743-52.

24. Darby I, Phan L, Post M. Periodontal health of dental clients in a community health setting. Australian Dental Jornal 2012; 57(4):486-92.

25. Bonotto DMV, Pintarelli TP, Santin G, Montes GR, Ferreira FM, Fraiz FC. Cárie dentária e gênero em adolescentes. Ver Odontol 2015; 20(2):

26. Júnior S, Aguiar NL, Barros WRC, Arantes DC, Nascimento LSD. Saúde Bucal do Adolescente: Revisão de Literatura. Adolesc Saúde (Online), 2016; 95-103.

\section{Endereço para correspondência:}

Samara Sandrelly de Moura Gomes

Rua Rodrigues Ferreira, 45, Várzea

Cep 50810-020. Recife-PE, Brasil

E-mail: samarasandrelly@yahoo.com.br

Recebido: 08/12/2016. Aceito: 03/02/2017. 